\title{
Erratum to: Expression in Pichia pastoris X33 of His-tagged lipase from a novel strain of Rhizopus oryzae and its mutant Asn 134 His: purification and characterization
}

\author{
Riadh Ben Salah
}

Published online: 3 January 2010

(C) Springer Science+Business Media B.V. 2009

Erratum to: World J Microbiol Biotechnol (2009) 25:1375-1384

DOI 10.1007/s11274-009-0024-4

The names of Ali Gargouri, Robert Verger, Youssef Gargouri, Hafedh Mejdoub should be removed from the above paper, as it was published without their permission.

The online version of the original article can be found under doi:10.1007/s11274-009-0024-4.

R. Ben Salah $(\varangle)$

Laboratoire de biochimie et de ge'nie enzymatique des lipases,

ENIS, Route de Soukra, "BPW", 3038 Sfax, Tunisia

e-mail: riadh_fss@yahoo.fr 